\title{
Inter-quartile analysis of resting heart rate and heart rate variability following spinal adjustment: A case study
}

\author{
John $\operatorname{Hart}^{1,2^{*}}$ \\ Correspondence: john.hart@purdueglobal.edu \\ ${ }^{1}$ Hart Chiropractic, Greenville, SC, USA. \\ ${ }^{2}$ Adjunct Faculty, Purdue University Global, USA.
}

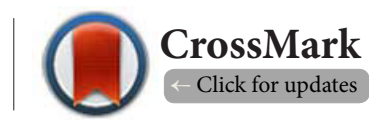

\begin{abstract}
Background: Different clinicians use heart rate tests to answer different questions. The medical doctor for example might use heart rate to diagnose a medical condition. One approach in chiropractic practice is to use heart rate information to assess the health of the autonomic nervous system. This retrospective case study uses outlier analysis for resting heart rate (HR) and resting heart rate variability (HRV) to assess the neurological efficacy of a single spinal adjustment for an individual patient.The purpose of the study is to show how basic statistical analysis can be used to assess neurological efficacy of a chiropractic adjustment on an individual patient basis.
\end{abstract}

Methods: HR and HRV were measured twice before the spinal adjustment, then once immediately following the adjustment and another measurement 2 weeks later. The four readings for each measure (HR and HRV) were analyzed using the inter-quartile method of outlier detection.

Results: The immediate post-adjustment findings for both measures remained essentially unchanged compared to the two pre-adjustment readings. However, the follow-up visit showed substantial improvement for both measures to the extent that they were shown to be statistical outliers.

Conclusion: This patient experienced statistically unusual autonomic improvement following a spinal adjustment according to HR and HRV, though the improvement was not measurable until 2 weeks later. Further study in other patients is planned.

Keywords: Chiropractic adjustment, resting heart rate, heart rate variability, biostatistics

\section{Introduction}

Different clinicians use heart rate information to answer different questions. The medical doctor for example might use heart rate data to determine the presence of a heart condition.

The traditional objective of chiropractic care is to improve neurological function by adjusting chiropractic subluxation $[1,2]$. In concept, a chiropractic subluxation is a slight vertebral misalignment that disturbs nearby nerves. A restored autonomic nervous system helps patients heal themselves of what ails them. Evidence-based methods such as resting heart rate variability $(\mathrm{HRV})$ and resting heart rate $(\mathrm{HR})$ help document whether neurological (autonomic) improvement has been accomplished [3].

Statistical analysis is typically used to compare groups of patients rather than an individual patient's pre-post findings. However, statistical analysis may be appropriate at the level of the individual patient if statistical assumptions are not violated. The benefit of such application is that the statistical results instantly pertain to that patient.

The present case study statistically analyzes HR and HRV before versus after a single spinal adjustment for an individual patient. Both of these measures (HR and HRV) are a good fit for the neurologically-focused chiropractic practice since they: a) are neurologically-based $[4,5]$ and b) have patient-centered outcomes research support (e.g., patients with healthier HR and HRV tend to live longer than counterparts who have less healthy $H R$ and $H R V)[5,6]$. Additionally, there is emerging evidence that these measures tend to improve following chiropractic care [7-9].

The purpose of the study is to show how basic statistical analysis can be applied to the autonomic assessments of HR and HRV to determine whether autonomic change following 
John Hart, Neuroscience Discovery 2019,

spinal adjustment is statistically unusual.

\section{Methods}

An adult male patient from the author's chiropractic practice signed an informed consent form for this retrospective case study. In addition, the study was declared Exempt by the Institutional Review Board at Purdue University Global.

In early 2019 the patient consulted the author for acute hip pain. Routine spinal exam was performed to determine if the patient had a spinal subluxation, conceptually defined as a slight misalignment that disturbs nerve function. The operational definition of subluxation in the study was based on the exam procedures at Hart Chiropractic that included: a) neurological assessment using HR and HRV and b) spinal alignment assessment using palpation. The neurological assessments were used for determining when to adjust; the location for adjustment was based on the author's upper cervical spine focus.

For HRV, the time domain measure of root mean square of successive differences between heart beats (rMSSD), measured in milliseconds (ms) was used. The terms rMSSD and HRV are used interchangeably in this paper. HR was measured in beats per minute (BPM).

Both measures (HR and HRV) were recorded using the Heart Rate Variability Logger app [10] in conjunction with the Kyto ear clip sensor [11] (Figure 1). The sensor sends a Bluetooth signal to the smartphone app and the set-up has good agreement with standard ECG technology $[11,12]$. Both measures (HR and HRV) were recorded simultaneously for 1 minute each recording session with the patient in the seated position after a seated rest period of at least 5 minutes.

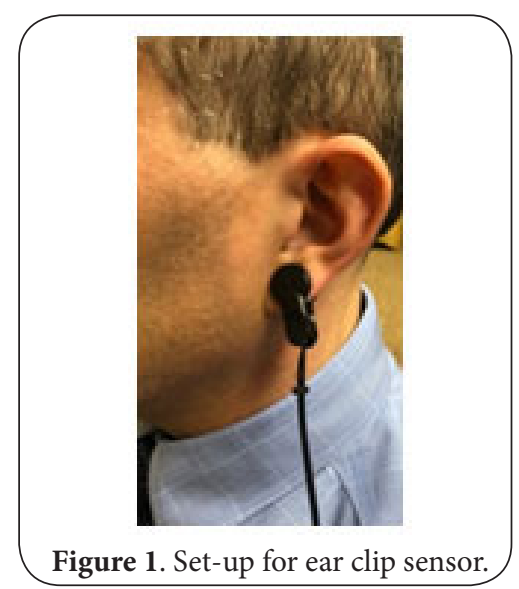

The study included two visits with one spinal adjustment - occurring on Visit 1. The recording for HR and HRV was performed: a) two times before the adjustment to establish a baseline; b) once approximately 5 minutes after the adjustment on this same visit (Visit 1); and c) once on the follow-up visit two weeks later (Visit 2). Both visits occurred during the same hour of the day and according to the patient no changes in lifestyle or medication were made between the two visits.

Normative data indicate that average HR for healthy adult males is approximately 70 BPM [13] though lower than this is of course neurologically healthier. For HRV, a healthy rMSSD value for adults is minimally, approximately $25 \mathrm{~ms}[\mathbf{1 4 , 1 5}$. In other words, a lower HR is neurologically healthier than a higher $\mathrm{HR}$, while a higher HRV is neurologically healthier than a lower HRV. A low HR indicates an autonomic nervous system that is less stressed (more parasympathetic and/or less sympathetic influenced) compared to higher HR. A higher HRV indicates an autonomic nervous system that is more adaptive - to the continuous changes occurring internally, even at rest.

\section{Statistical analysis}

To determine whether any pre-post improvement could be considered statistically unusual, the four readings (three from Visit 1+ one from Visit 2) were analyzed in Excel by the author using the inter-quartile method of outlier detection [16]. The method used the conventional factor of 1.5 for identifying potential moderate outliers, multiplying it by the interquartile range (IQR) to obtain the fence limits:

$$
\begin{aligned}
& \text { Lower fence }=\text { Quartile } 1-(1.5 * \text { IQR }) \\
& \text { Upper fence }=\text { Quartile } 3+(1.5 * \text { IQR })
\end{aligned}
$$

Observed values outside the fences are considered outliers (statistically unusual findings).

\section{Results \\ Visit 1}

\section{Pre-adjustment 1}

HR was 85.6 BPM and HRV was 19.0 ms (Figure 2) both of which

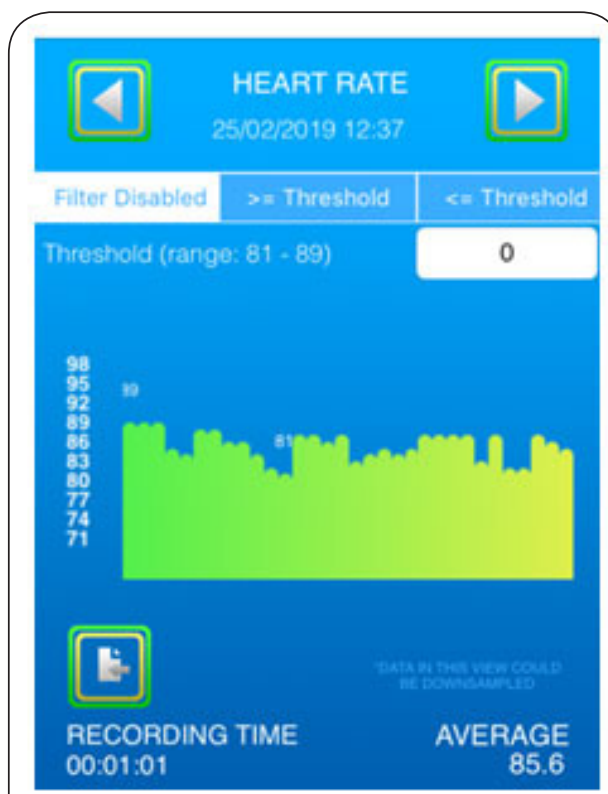

Figure 2. Visit 1, app screenshot for pre-adjustment reading \#1. 
were considered neurologically unhealthy. In the screenshots, more bumpiness indicates more variability, substantiated by the numerical HRV value.

\section{Pre-adjustment 2}

HR was 85.2 BPM and HRV was 19.1 ms (Figure 3), both of which are essentially the same as the pre 1 results and established a baseline of neurological disturbance. This disturbance along with a palpated misalignment of atlas (C1) vertebra led to a diagnosis of atlas subluxation.

The subluxation was adjusted by the author with a percussion type instrument while the patient remained seated (set-up example shown in Figure 4).

\section{Post-adjustment}

Approximately 5 minutes following the adjustment, $\mathrm{HR}$ was 85.2 BPM and HRV was 20.9 ms (Figure 5), still essentially the same as both pre-adjustment readings for both measures. This indicated that neurological benefit was either not achieved or not yet measurable due to the body still processing the adjustment.

\section{Visit 2 (15 days later)}

The patient's hip symptom improved between visits. However, he was now having new symptoms - in the neck and low back areas, that began approximately two days prior to this visit (Visit 2). HR decreased (improved) by approximately 20 beats to $66.5 \mathrm{BPM}$ and HRV increased (improved) by about $15 \mathrm{~ms}$ to $35.9 \mathrm{~ms}$ (Figure 6). Both improvements were large enough to be detected as outliers (Figures 7-8, Table 1).

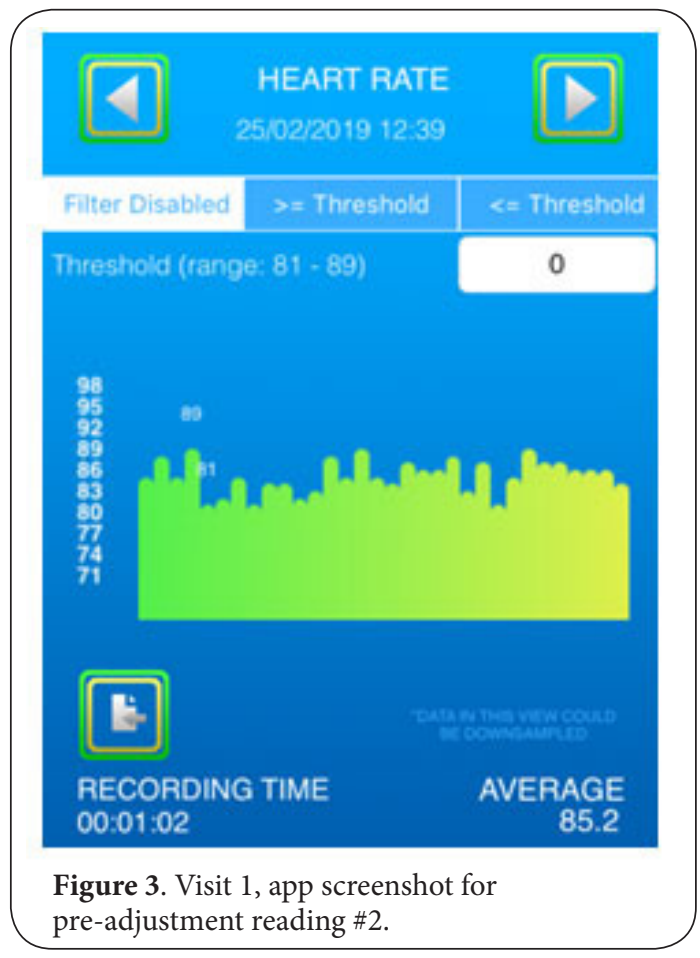

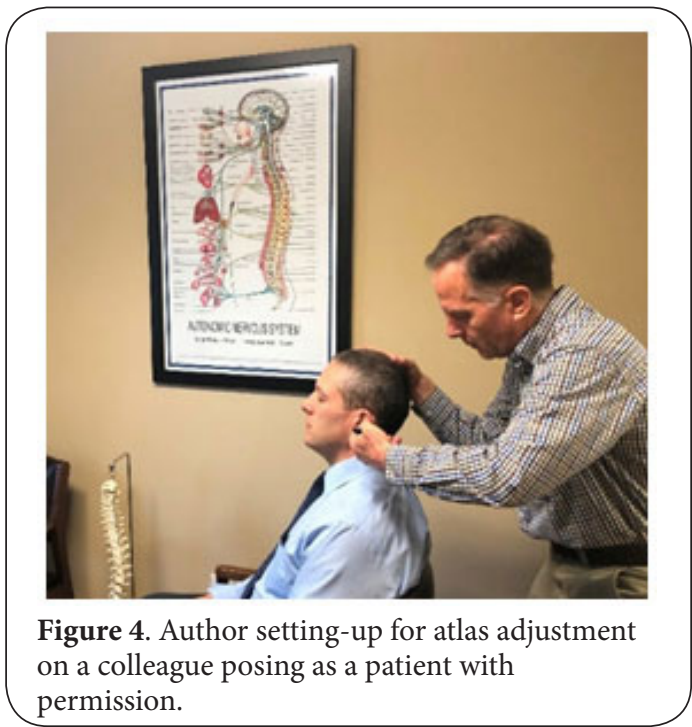

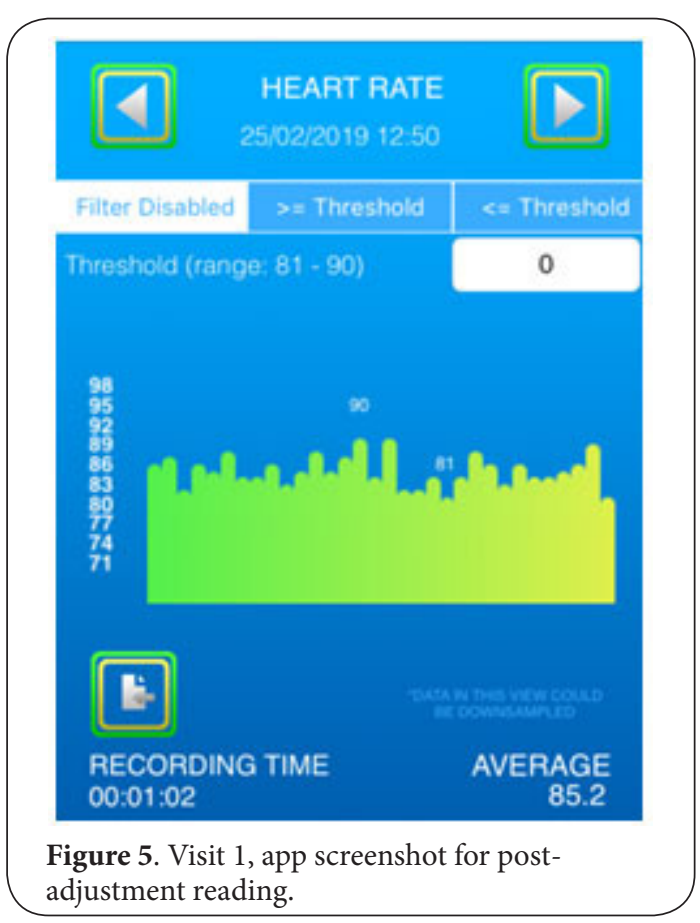

Consequently, no adjustment was indicated this visit because of the autonomic improvement.

\section{Discussion}

The immediate post-adjustment reading on Visit 1 were essentially unchanged compared to that visit's pre-adjustment readings for both HR and HRV. However, the follow-up visit (Visit 2, 15 days later) did show a change in the form of improved HR and HRV. Moreover, these improvements were large enough to be detected as outliers.

It was theorized on Visit 2 that the neck and low back symptoms were part of an internal realignment process, particularly 


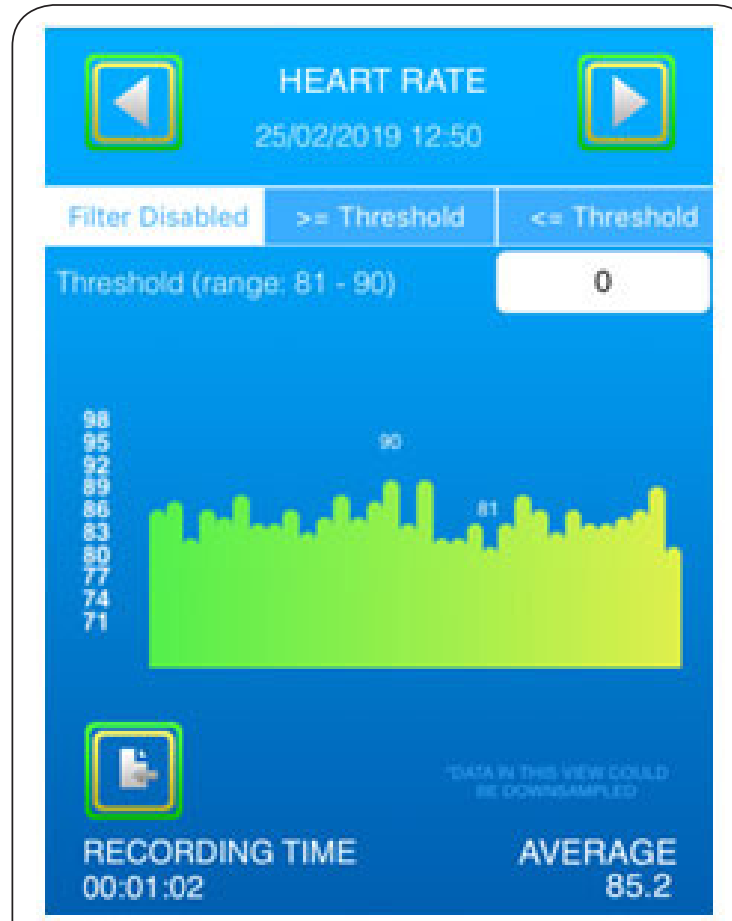

Figure 6. Visit 2 app screenshot.
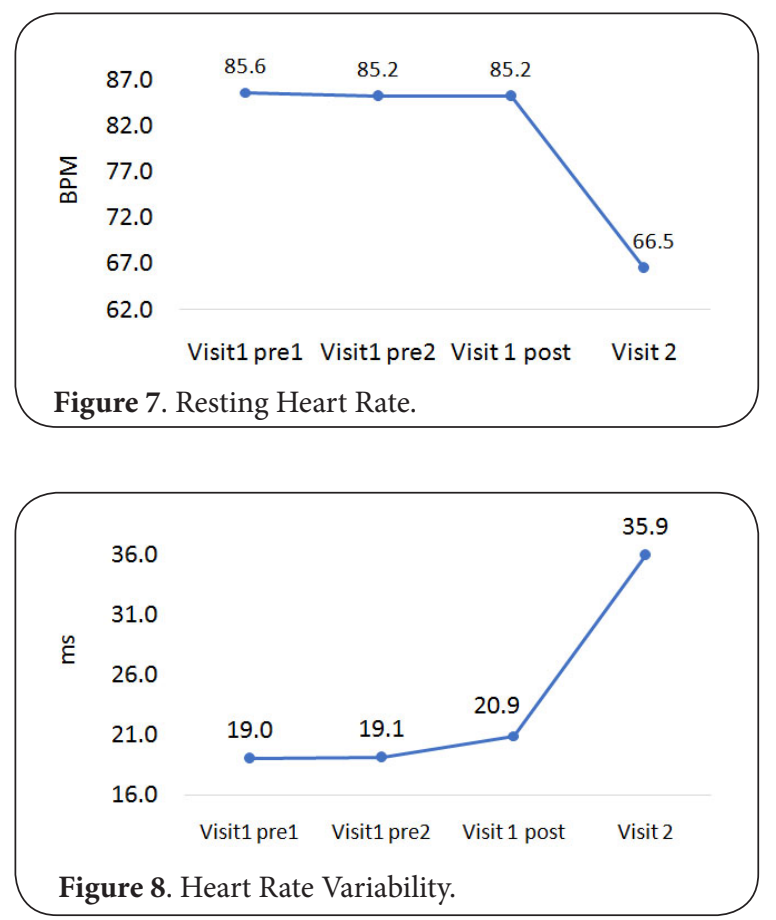

Table 1. Summary Statistics.

\begin{tabular}{lllllll} 
Test & Visit 1 pre 1 & Visit 1 pre 2 & Visit 1 post & Visit 2 & LF & UF \\
\hline HR $(B P M)$ & 85.6 & 85.2 & 85.2 & $66.5^{*}$ & 73.4 & 92.5 \\
HRV $(\mathrm{ms})$ & 19.0 & 19.1 & 20.9 & $35.9^{*}$ & 10.7 & 33.0 \\
\hline LF and UF = lower and upper fence in outlier analysis. \\
* Value calculated as an outlier since it is outside the fences.
\end{tabular}

when the neurological findings had substantially improved.

Adjustment was not given on Visit 2 due to the improved autonomic indicators of HR and HRV. Yes, the patient was having what could be called "chiropractic symptoms" but the risk of intervention when not neurologically indicated could adversely affect autonomic health [17]. This risk was considered, and the author opted to avoid the risk. A few months after Visit 2, the patient sent an unsolicited message to the author stating he was having no pain and "doing so well."

\section{Limitations}

It could be theorized that the resolution of the initial symptom on Visit 2 might be responsible for the improved HR and HRV. However, on that visit (Visit 2) the patient was still having pain, just in different locations.

In a similar case study on another patient by this author, HRV improvement was detectable on the same visit as the adjustment [18]. That study, considered along with the present study suggests, unsurprisingly that different patients may show improvements at different times following their adjustment. In the other study [18] the outlier was detected using Grubbs test, which requires a normal distribution. Data in the present study showed marked departures from normal distribution according to normal probability plot assessment (for both HR and HRV). Consequently, the inter-quartile method, which does not strictly require a normal distribution [19], was considered appropriate for data in the present study.

Other limitations include those that typically accompany observational studies such as this, e.g., limited generalizability. Although no claim of cause-and-effect can be made in case studies, the improved findings 2 weeks after the adjustment do appear to be related to the adjustment because: a) the rather strong baseline showing neurological disturbance was followed by marked improvement, though 2 weeks later; and b) no other life style changes were made by the patient between the visits.

\section{Conclusion}

This study provides an example of how basic statistics can be applied to the level of the individual patient for determining whether autonomic change following a spinal adjustment is statistically unusual. In this case, the patient experienced substantial neurological improvement approximately 2 weeks following spinal adjustment, evidenced by outlier analysis of autonomic assessments. Further study in other patients is planned.

\section{Competing interests}

The author declares that he has no competing interests.

\section{Acknowledgement}

The author thanks the patient for his permission to publish his case. 
John Hart, Neuroscience Discovery 2019,

\section{Publication history}

Editor: Shiwei Duan, Ningbo University, China.

Received: 05-Nov-2019 Final Revised: 08-Dec-2019

Accepted: 10-Dec-2019 Published: 21-Dec-2019

\section{References}

1. Palmer College of Chiropractic. Philosophy Statement. 2019 | Website

2. Association of Chiropractic Colleges (ACC). Chiropractic paradigm. 2019 I Website

3. Hart J. Association between heart rate variability and manual pulse rate. J Can Chiropr Assoc. 2013; 57:243-50. | PubMed Abstract | PubMed FullText

4. Mensink GB and Hoffmeister $H$. The relationship between resting heart rate and all-cause, cardiovascular and cancer mortality. Eur Heart J. 1997; 18:1404-10. | Article | PubMed

5. Zulfiqar U, Jurivich $D A$, Gao W and Singer DH. Relation of high heart rate variability to healthy longevity. Am J Cardiol. 2010; 105:1181-5. | Article I PubMed

6. Zhang $G Q$ and Zhang W. Heart rate, lifespan, and mortality risk. Ageing Res Rev. 2009; 8:52-60. | Article | PubMed

7. Zhang J, Dean D, Nosco D, Strathopulos D and Floros M. Effect of chiropractic care on heart rate variability and pain in a multisite clinical study. J Manipulative Physiol Ther. 2006; 29:267-74. | Article | PubMed

8. Hart J. Reduction of resting pulse rate following chiropractic adjustment of atlas subluxation. Annals of Vertebral Subluxation Research. 2014; 3:16-21.

9. Win NN, Jorgensen AM, Chen YS and Haneline MT. Effects of Upper and Lower Cervical Spinal Manipulative Therapy on Blood Pressure and Heart Rate Variability in Volunteers and Patients With Neck Pain: A Randomized Controlled, Cross-Over, Preliminary Study. J Chiropr Med. 2015; 14:1-9. | Article | PubMed Abstract | PubMed FullText

10. Altini M. Heart rate variability Logger - app details. 2013. | Website

11. Vescio B, Salsone M, Gambardella A and Quattrone A. Comparison between Electrocardiographic and Earlobe Pulse Photoplethysmographic Detection for Evaluating Heart Rate Variability in Healthy Subjects in Short- and Long-Term Recordings. Sensors (Basel). 2018; 18. | Article | PubMed Abstract | PubMed FullText

12. Plews DJ, Scott B, Altini M, Wood M, Kilding AE and Laursen PB. Comparison of heart rate variability recording with smart phone photoplethysmographic Polar H7 chest strap and electrocardiogram methods. Int J Sport Physiol. 2017; 12:1-17.

13. Ostchega Y, Porter KS, Hughes J, Dillon CF and Nwankwo T. Resting pulse rate reference data for children, adolescents, and adults: United States, 1999-2008. Natl Health Stat Report. 2011; 1-16. I Pdf I PubMed

14. Baek HJ, Cho CH, Cho J and Woo JM. Reliability of ultra-short-term analysis as a surrogate of standard 5-min analysis of heart rate variability. Telemed J E Health. 2015; 21:404-14. | Article | PubMed

15. Kim GM and Woo JM. Determinants for heart rate variability in a normal Korean population. J Korean Med Sci. 2011; 26:1293-8. I Article | PubMed Abstract | PubMed FullText

16. Devore J and Peck R. Statistics: The exploration of data. 4th Edition. Pacific Grove, CA: Duxbury. 2001.

17. Hart J. Optimal level of heart rate variability for spinal adjustment: A case report. Journal of Contemporary Chiropractic. 2019; 2:103-108. | Article

18. Hart J. Improvement in heart rate variability following spinal adjustment: A case study in statistical methodology for a single office visit. Int J Stats Med Res. 2019; 8:17-22.

19. Brownlee J. How to use statistics to identify outliers in data. Machine Learning Mastery. 2019. I Website
Citation:

Hart J. Inter-quartile analysis of resting heart rate and heart rate variability following spinal adjustment: A case study. Neurosci Discov. 2019; 7:1.

http://dx.doi.org/10.7243/2052-6946-7-1 